\title{
Review
}

Ophthalmic

Research

Ophthalmic Res 2016;55:172-179

DOI: $10.1159 / 000443627$
Received: September 22, 2015

Accepted after revision: December 21, 2015

Published online: February 13, 2016

\section{Association of MMP-9 Gene Polymorphisms with Glaucoma: A Meta-Analysis}

\author{
Yiqun Zhang $^{\text {a }}$ Mingjie Wang ${ }^{b}$ Sunyi Zhang ${ }^{\text {a }}$ \\ a Department of Ophthalmology, Affiliated Hospital of Suzhou University, Changzhou No. 4 People's Hospital, \\ Changzhou, and ${ }^{\mathrm{b}}$ Nanjing Medical University, Nanjing, China
}

\section{Key Words}

Glaucoma · Meta-analysis · MMP-9 · Polymorphisms ·

Single-nucleotide polymorphisms

\begin{abstract}
The aim of this study was to evaluate the associations between matrix metalloproteinase-9 (MMP-9) gene polymorphisms (rs17576 and rs3918249) and glaucoma risk. All eligible studies were searched in PubMed, Embase, the Cochrane Library and the China Knowledge Resource Integrated Database. Pooled odds ratios and $95 \%$ confidence intervals were used to assess associations between MMP-9 gene polymorphisms and glaucoma. Seven studies on rs $17576(1,357$ cases and 1,432 controls) and 3 studies on rs3918249 (550 cases and 794 controls) were included. The results suggest that rs 17576 was not associated with glaucoma risk based on current publications. However, stratification analyses indicated that GG genotypes increased the risk of primary open-angle glaucoma in a recessive model (GG vs. AA + AG). The rs3918249 polymorphism was also associated with a decreased risk of glaucoma, especially for Caucasian patients. To sum up, our data indicate that rs 17576 polymorphism is not related to glaucoma and rs3918249 polymorphism might be a protective factor against glaucoma.
\end{abstract}

\section{Introduction}

Glaucoma, characterized by a gradual degeneration of retinal ganglion cells (RGCs) and progressive degeneration of the optic nerve [1], is the second leading cause of irreversible blindness and expected to affect approximately 80 million people worldwide by 2020 [2]. Glaucoma includes two main types: primary open-angle glaucoma (POAG) and primary angle closure glaucoma (PACG). The pathogenesis of glaucoma remains unclear. Various risk factors, including age, variable susceptibility of the optic nerve, elevated intraocular pressure (IOP), diabetes, vascular factors, myopia, cigarette smoking and a positive family history, have been associated with glaucoma [3]. A positive family history significantly increases the risk of glaucoma [4], with genetic factors potentially involved in its etiology. Furthermore, many studies have reported a link between matrix metalloproteinase-9 (MMP-9) gene polymorphisms and glaucoma [5-7].

MMP-9, also known as $92-\mathrm{kDa}$ gelatinase or type $\mathrm{V}$ collagenase, is located on chromosome 20q11.2-q13.1 [8]. MMP-9 encodes a multidomain enzyme, which is actively involved in extracellular matrix (ECM) remodeling

Yiqun Zhang and Mingjie Wang contributed equally to this study. 
in glaucoma patients $[7,9]$. The trabecular meshwork (TM) is thought to be the main site of aqueous outflow resistance in normal subjects and patients with glaucoma [10]. Some studies have reported that intraocular MMP-9 expression is increased in the aqueous humor outflow tract of glaucomatous eyes $[11,12]$. Many studies have suggested that the changed structure and accumulation of ECM in the TM increase TM resistance [13]. Aqueous outflow resistance depends on a complicated equilibrium of ECM biosynthesis versus proteolysis, and changes might be due to qualitative alterations in ECM composition [14]. More importantly, MMP-9 has been suggested to be closely associated with accumulation of ECM in the TM [13]. This remodeling within the TM results in elevated IOP in glaucoma patients [15]. Ultrastructural examinations of TM in POAG patients suggested alterations in ECM composition and structure [14]. Therefore, a role of $M M P-9$ in the pathogenesis of glaucoma has been strongly suggested. Single-nucleotide polymorphisms (SNPs) in the MMP-9 gene may alter the function of MMP-9, thus affecting ECM restoration. Theoretically, we hypothesized a potential relationship between glaucoma and MMP-9 gene polymorphisms, and recent studies indicated that rs 17576 and rs3918249, the two SNPs of MMP-9, might be associated with glaucoma susceptibility [5-7, 16-20]. However, the results remain controversial, with some studies unable to identify any association between $M M P-9$ gene polymorphisms and glaucoma. In the following study, a meta-analysis was performed to evaluate the contradictory results from these relevant studies and to clarify the associations between rs 17576 and rs3918249 polymorphisms and glaucoma.

\section{Methods}

\section{Literature Search}

We performed a comprehensive search in PubMed, Embase, the Cochrane Library and the China Knowledge Resource Integrated Database to identify studies through June 5, 2015, that were related with MMP-9 gene polymorphisms and glaucoma. The following search terms were used: 'glaucoma', 'primary open angle glaucoma', 'POAG', 'primary angle closure glaucoma', 'PACG', 'primary angle closure', 'PAC', 'MMP9', 'MMP-9', 'matrix metalloproteinase 9' and 'polymorphism'. Two independent investigators conducted the search. No language or any restrictions were placed on the search. We also searched the reference lists of all related studies to identify other initially omitted studies. Any disagreements were resolved by consensus.

Inclusion and Exclusion Criteria

Inclusion criteria were: (1) evaluation of the associations between MMP-9 gene polymorphisms (rs17576 and rs3918249) and glaucoma; (2) human studies; (3) sufficient data for estimating the odds ratios (ORs) and 95\% confidence intervals (CIs), and (4) independent case-control studies. Exclusion criteria were: (1) a duplication of previous publications; (2) a review, editorial or other nonoriginal study; (3) a family-based study of pedigrees; (4) studies without detailed genotype data, and (5) inclusion of subjects with other diseases that might influence the results.

\section{Data Extraction}

For all eligible studies, the extracted information included the name of the first author, the publication year, the numbers of cases and controls, the country of origin, the ethnicity, the genotyping method, the $\mathrm{p}$ value for Hardy-Weinberg equilibrium (HWE), and the $M M P-9$ genotype frequencies in cases and controls. Extraction of the data was independently performed by two authors who checked the data extracted and agreed on all values; disagreements were resolved by discussion.

\section{Quality Assessment}

Two reviewers independently evaluated the study quality based on the Newcastle-Ottawa Scale (NOS) [21]. The NOS criteria included three aspects: (1) subject selection (from 0 to 4 ); (2) the comparability of the subject (from 0 to 2), and (3) the clinical outcome (from 0 to 3 ). Total NOS scores ranged from 0 to 9 . A score of 5-9 stars is considered to be of generally high methodological quality, whereas a score of $0-4$ is regarded as relatively poor in quality [22]. All included studies were screened for quality assessment by two researchers. Any disagreement was resolved by a comprehensive reassessment by a third researcher until consensus was reached.

\section{Statistical Analyses}

All statistical analyses were performed using the Stata 11.0 software (StataCorp, College Station, Tex., USA). Pooled ORs and 95\% CIs were calculated to evaluate the strength of the associations between the MMP-9 gene polymorphisms (rs17576 and rs3918249) and glaucoma. Heterogeneity was evaluated by the Q statistic (significant at $\mathrm{p}<0.1)$ and $\mathrm{I}^{2}$ statistic $\left(\mathrm{I}^{2}>50 \%\right.$ indicating significant heterogeneity) [23]. If no significant heterogeneity was detected among the studies, the fixed-effect model was used; otherwise, the random-effect model was used. Pooled ORs were calculated for the allele contrast, the dominant model, the recessive model, the homozygous model and the heterozygous model. We performed sensitivity analyses by omitting each study in turn to determine the effect on the test of heterogeneity and to evaluate the stability of the overall results. Potential publication bias was evaluated using Begg's and Egger's linear regression tests [24]. $\mathrm{p}<0.05$ suggested significant publication bias.

\section{Results}

\section{Characteristics of the Published Studies}

Our initial search yielded 55 studies. After removing duplicates and screening the titles and abstracts, 45 studies were removed. After reviewing the full text, 2 studies $[25,26]$ were excluded, because they did not conform to the inclusion criteria. We finally identified 8 eligible stud- 
ies in this meta-analysis [5-7, 16-20]. Figure 1 summarizes the selection of eligible studies for the meta-analysis. Seven studies (1,357 cases and 1,432 controls) were included to determine the association between glaucoma and the rs17576 polymorphism [5-7, 17-20], and 3 studies (550 cases and 794 controls) for the rs 3918249 polymorphism $[6,16,17]$. Two studies investigated both rs17576 and rs3918249 polymorphisms [6, 17]. These studies were published from 2006 to 2014 . Genotype distributions of the controls in these studies all conformed to the HWE. The NOS scores of all included studies ranged from 6 to 7 stars, indicating that they were studies of high methodological quality. More detailed characteristics of the included studies are summarized in table 1 .

Meta-Analysis: rs17576 Polymorphism and Glaucoma

A random-effect model was used for all five models including the allele model ( $G$ vs. A), recessive model (GG vs. $A A+A G)$, dominant model $(G G+A G$ vs. AA), heterozygous model (AG vs. AA) and homozygous model (GG vs. AA), indicating that these included studies had significant heterogeneity. As shown in table 2, the rs 17576 polymorphism was not associated with glaucoma in any of the five models ( $\mathrm{G}$ vs. A: OR, 0.98; 95\% CI, 0.68-1.41, $\mathrm{p}=0.925$; fig. 2). Stratification analyses were conducted according to ethnicity and the type of glaucoma (table 3 ). Our data indicated that the rs 17576 polymorphism was significantly associated with POAG (GG vs. AA + AG: OR, 1.53; 95\% CI, 1.03-2.25, $\mathrm{p}=0.034$; fig. 3). However, we did not find that rs17576 was associated with Asian or Caucasian ethnicity when we conducted stratification analyses of ethnicities. We assessed sensitivity by omitting each study one at a time in every genetic model. The pooled ORs for the association between the rs 17576 polymorphism and the glaucoma risk indicated that our data were stable and dependable. Both Egger's and Begg's tests were used to evaluate the publication bias of this study. The result suggested that there was no obvious publication bias in overall analyses for the rs17576 polymorphism (G vs. A, $p_{\text {begg }}=0.764$ and $p_{\text {egger }}=0.374 ; \mathrm{GG}+\mathrm{AG}$ vs. $A A, p_{\text {begg }}=0.548$ and $p_{\text {egger }}=0.364 ; \mathrm{GG}$ vs. $A A+A G$, $\mathrm{p}_{\text {begg }}=0.548$ and $\mathrm{p}_{\text {egger }}=0.960 ; \mathrm{GG}$ vs. $\mathrm{AA}, \mathrm{p}_{\text {begg }}=0.368$ and $\mathrm{p}_{\text {egger }}=0.259 ; \mathrm{AG}$ vs. $\mathrm{AA}, \mathrm{p}_{\text {begg }}=0.230$ and $\mathrm{p}_{\text {egger }}=$ $0.290)$.

\section{Meta-Analysis: rs3918249 Polymorphism and Glaucoma}

A fixed-effect model was conducted for the recessive model (TT vs. CC + CT), heterozygous model (CT vs. CC) and homozygous model (TT vs. CC), and a random-

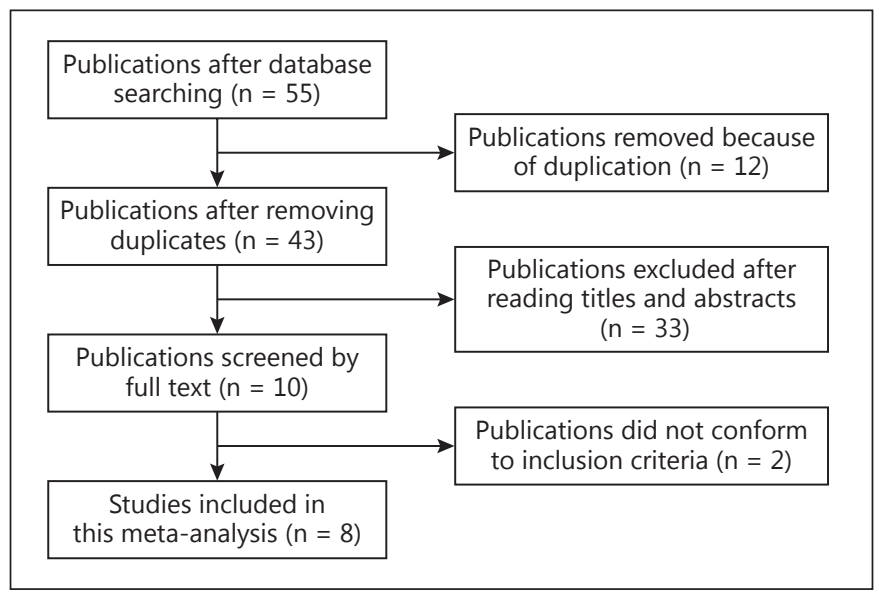

Fig. 1. Selection for eligible studies included in this meta-analysis.

effect model was used for the allele model ( $\mathrm{T}$ vs. C) and dominant model (TT + CT vs. CC). The results indicated that the rs3918249 polymorphism might be a protective factor for glaucoma patients (TT vs. CC + CT: OR, 0.71; 95\% CI, 0.51-0.98, p = 0.040; fig. 4; table 2). Stratification analyses also suggested that the rs3918249 polymorphism decreased the risk of glaucoma, especially in Caucasian glaucoma patients. Sensitivity analysis indicated that our data were stable and dependable. For the rs3918249 polymorphism, the $\mathrm{p}$ value of Egger's and Begg's tests indicated that there was no evident publication bias ( $\mathrm{T}$ vs. C, $\mathrm{p}_{\text {begg }}=1.000$ and $\mathrm{p}_{\text {egger }}=0.548 ; \mathrm{TT}+\mathrm{CT}$ vs. $\mathrm{CC}, \mathrm{p}_{\text {begg }}=$ 1.000 and $\mathrm{p}_{\text {egger }}=0.548 ;$ TT vs. $\mathrm{CC}+\mathrm{CT}, \mathrm{p}_{\text {begg }}=0.296$ and $\mathrm{p}_{\text {egger }}=0.180 ; \mathrm{CT}$ vs. $\mathrm{CC}, \mathrm{p}_{\text {begg }}=1.000$ and $\mathrm{p}_{\text {egger }}=0.629$, and TT vs. $C C$, $p_{\text {begg }}=1.000$ and $\left.p_{\text {egger }}=0.776\right)$.

\section{Discussion}

This is the first meta-analysis to summarize the association between MMP-9 gene polymorphisms and glaucoma susceptibility. Our data suggest that rs17576 is not associated with the glaucoma risk. However, stratification analyses indicated that rs 17576 increased the risk of POAG. Our data also showed that rs3918249 was associated with a decreased risk of glaucoma in Caucasians.

MMPs comprise a family of at least 25 secreted zinc proteinases [27]. It has been reported that enhanced activation of MMPs, such as MMP-9, may play an important role in the ECM-remodeling process [9]. PACG patients commonly present biometric ocular characteristics, such as increased thickness of the lens [28], a shallow anterior 
Table 1. Characteristics of included studies

\begin{tabular}{|c|c|c|c|c|c|c|c|c|c|c|c|c|c|}
\hline \multirow[t]{2}{*}{ Study ID } & \multirow[t]{2}{*}{ Country } & \multirow[t]{2}{*}{ Ethnicity } & \multicolumn{3}{|c|}{ Cases } & \multicolumn{3}{|c|}{ Controls } & \multicolumn{2}{|l|}{ Allele } & \multirow[t]{3}{*}{ HWE } & \multirow{2}{*}{$\begin{array}{l}\text { Genotyping } \\
\text { method }\end{array}$} & \multirow[t]{2}{*}{ QAS } \\
\hline & & & & & & & & & cases/controls & cases/controls & & & \\
\hline rs 17576 & & & AA & $\mathrm{AG}$ & GG & AA & AG & GG & A & G & & & \\
\hline Micheal [5], 2013 & Pakistan & Asian & 41 & 80 & 73 & 40 & 53 & 25 & $162 / 133$ & $226 / 103$ & 0.353 & PCR & 6 \\
\hline Shi [17], 2013 & China & Asian & 20 & 78 & 133 & 29 & 113 & 164 & $118 / 171$ & $344 / 441$ & 0.156 & PCR & 7 \\
\hline Awadalla [6], 2011 & Australia & Caucasian & 37 & 49 & 18 & 132 & 109 & 27 & $123 / 373$ & $85 / 163$ & 0.563 & PCR & 6 \\
\hline Mossbock [18], 2010 & Australia & Caucasian & 139 & 141 & 42 & 102 & 120 & 26 & $419 / 324$ & $225 / 172$ & 0.328 & PCR & 6 \\
\hline Cong [19], 2009 & China & Asian & 16 & 76 & 119 & 6 & 75 & 124 & $108 / 87$ & $314 / 323$ & 0.215 & PCR & 6 \\
\hline Aung [20], 2008 & Singapore & Asian & 13 & 69 & 135 & 4 & 31 & 48 & $95 / 39$ & $339 / 127$ & 1.000 & PCR & 7 \\
\hline Wang [7], 2006 & Taiwan & Asian & 44 & 17 & 17 & 15 & 33 & 38 & $105 / 63$ & $51 / 109$ & 0.108 & PCR & 6 \\
\hline rs3918249 & & & $\mathrm{CC}$ & CT & $\mathrm{TT}$ & $\mathrm{CC}$ & $\mathrm{CT}$ & $\mathrm{TT}$ & $\mathrm{C}$ & $\mathrm{T}$ & & & \\
\hline Gao [16], 2014 & China & Asian & 91 & 109 & 13 & 104 & 103 & 14 & $291 / 311$ & $135 / 131$ & 0.106 & PCR-RFLP & 6 \\
\hline Shi [17], 2013 & China & Asian & 133 & 79 & 19 & 160 & 116 & 30 & $345 / 436$ & $117 / 176$ & 0.209 & PCR & 7 \\
\hline Awadalla [6], 2011 & Australia & Caucasian & 19 & 49 & 38 & 27 & 109 & 131 & $87 / 163$ & $125 / 371$ & 0.564 & PCR & 7 \\
\hline
\end{tabular}

QAS = Quality assessment score; HWE = Hardy-Weinberg equilibrium.

Table 2. Meta-analysis of the association between MMP-9 gene polymorphisms and glaucoma susceptibility

\begin{tabular}{|c|c|c|c|c|c|}
\hline Genetic contrasts & Random-/fixed-effect model & OR (95\% CI) & $\mathrm{p}$ value & $\mathrm{I}^{2}, \%$ & $\mathrm{p}$ for heterogeneity \\
\hline \multicolumn{6}{|l|}{ rs 17576} \\
\hline G vs. A & random & $0.98(0.68,1.41)$ & 0.925 & 88.5 & 0.001 \\
\hline GG vs. $A A+A G$ & random & $1.14(0.79,1.64)$ & 0.482 & 73.2 & 0.001 \\
\hline AG vs. AA & random & $0.77(0.47,1.28)$ & 0.319 & 77.3 & 0.001 \\
\hline GG vs. AA & random & $0.93(0.45,1.91)$ & 0.834 & 85.5 & 0.001 \\
\hline T vs. C & random & $0.84(0.62,1.14)$ & 0.267 & 67.9 & 0.045 \\
\hline TT + CT vs. CC & random & $0.84(0.56,1.27)$ & 0.404 & 63.1 & 0.066 \\
\hline TT vs. $\mathrm{CC}+\mathrm{CT}$ & fixed & $0.71(0.51,0.98)$ & 0.040 & 0.0 & 0.462 \\
\hline CT vs. CC & fixed & $0.93(0.3,1.19)$ & 0.564 & 41.0 & 0.184 \\
\hline TT vs. CC & fixed & $0.68(0.46,1.02)$ & 0.061 & 39.9 & 0.189 \\
\hline
\end{tabular}

Only TT vs. CC + CT showed a statistically significant difference (italics; $\mathrm{p}<0.05$ ).

chamber [29] and a short axial length [30]. ECM remodeling was shown to be a pivotal factor for short axial length in small eyes [19]. The short axial length in PACG patients may alter MMP-9 activity in ECM remodeling during ocular development [7] due to variants within or near this gene. The ECM of TM plays an important role in regulating IOP in glaucomatous eyes. Increases in the trabecular outflow resistance result in elevated IOP in POAG [15], which is the main risk factor for glaucomatous optic neuropathy. Bradley et al. [31] reported that injection of MMP-9 in a human outflow model led to a significantly increased outflow facility. Babizhayev and Brodskaya [13] suggested that MMP-9 was related to ECM accumulation and structural ECM changes in the TM, which changed the resistance to TM and IOP in POAG patients. A significantly increased level of MMPs (including MMP-9) in the aqueous humor of patients with POAG has been reported previously [32].

Glaucomatous damage to the optic nerve and retina of patients is often caused by pathological IOP elevation [33]. Loss of RGCs is the final end point of glaucoma. Chintala et al. [34] reported that apoptosis of RGCs cor- 


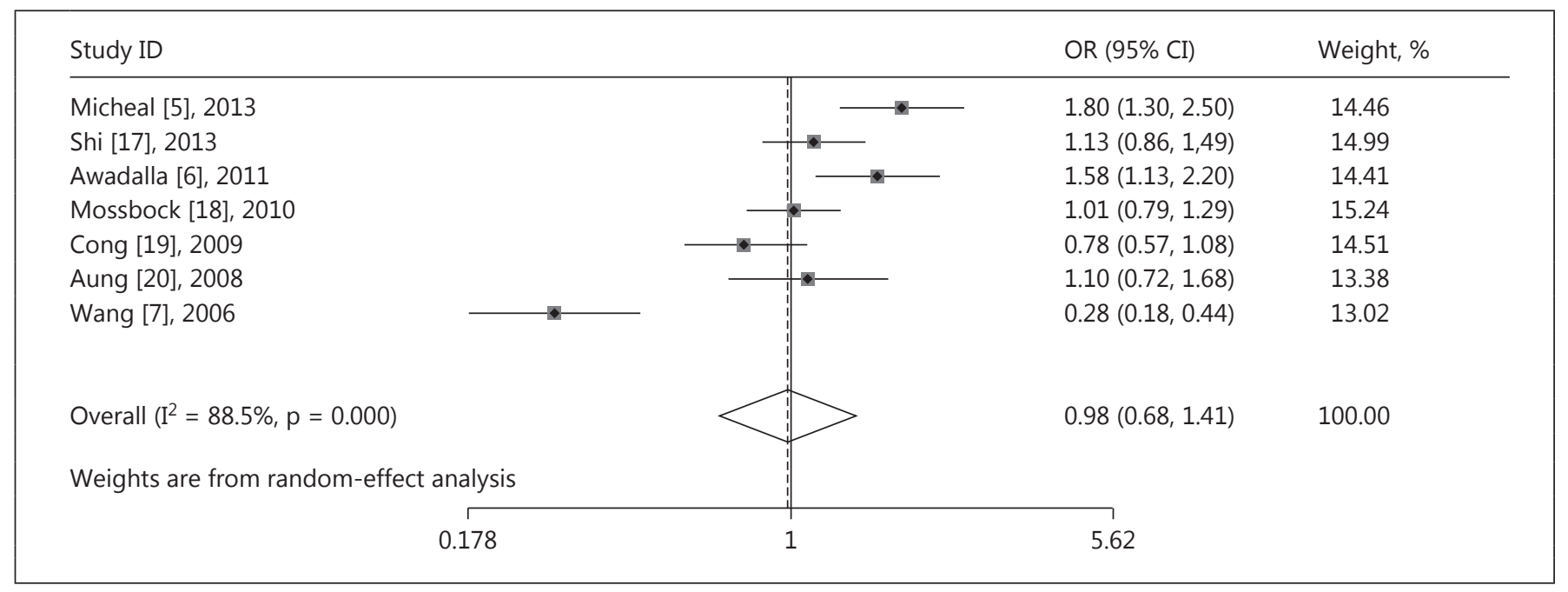

Fig. 2. Forest plot showing ORs for the association between the $M M P-9$ gene rs 17576 polymorphism and the risk of glaucoma (allele model: G vs. A).

Table 3. Stratified analyses between the $M M P-9$ gene polymorphisms and risk of glaucoma

\begin{tabular}{|c|c|c|c|c|c|c|c|c|}
\hline \multirow[t]{2}{*}{ Variable } & \multicolumn{4}{|c|}{ rs17576, cases/controls } & \multicolumn{4}{|l|}{ OR (95\% CI) } \\
\hline & AA & AG & GG & G vs. A & $\mathrm{GG}+\mathrm{AG}$ vs. AA & GG vs. $A A+A G$ & AG vs. AA & GG vs. AA \\
\hline \multicolumn{9}{|l|}{ Ethnicity } \\
\hline \multirow{2}{*}{$\begin{array}{c}\text { Caucasian } \\
\text { p value }\end{array}$} & $176 / 234$ & $190 / 229$ & $60 / 53$ & $1.25(0.81,1.93)$ & $1.24(0.66,2.34)$ & $1.49(0.99,2.23)$ & $1.15(0.63,2.10)$ & $1.62(0.82,3.20)$ \\
\hline & & & & 0.323 & 0.498 & 0.055 & 0.659 & 0.163 \\
\hline \multirow{2}{*}{$\begin{array}{l}\text { Asian } \\
\qquad \text { p value }\end{array}$} & $134 / 134$ & $320 / 358$ & $477 / 424$ & $0.88(0.52,1.49)$ & $0.64(0.25,1.66)$ & $1.02(0.64,1.62)$ & $0.61(0.27,1.34)$ & $0.71(0.25,2.04)$ \\
\hline & & & & 0.634 & 0.364 & 0.935 & 0.215 & 0.521 \\
\hline \multicolumn{9}{|c|}{ Type of glaucoma } \\
\hline \multirow{2}{*}{$\begin{array}{l}\text { POAG } \\
\text { p value }\end{array}$} & $165 / 142$ & $189 / 173$ & $80 / 51$ & $1.25(0.80,1.95)$ & $1.19(0.66,2.15)$ & $1.53(1.03,2.25)$ & $1.02(0.65,1.59)$ & $1.60(0.83,3.11)$ \\
\hline & & & & 0.336 & 0.564 & 0.034 & 0.937 & 0.162 \\
\hline \multirow{4}{*}{$\begin{array}{l}\text { PACG } \\
\text { p value }\end{array}$} & $145 / 226$ & $321 / 414$ & $457 / 426$ & $0.99(0.62,1.60)$ & $0.79(0.35,1.80)$ & $1.14(0.73,1.78)$ & $0.73(0.36,1.49)$ & $0.91(0.35,2.35)$ \\
\hline & & & & 0.981 & 0.579 & 0.569 & 0.390 & 0.840 \\
\hline & rs391824 & , cases/con & trols & & OR $(95 \%$ CI $)$ & & & \\
\hline & $\mathrm{CC}$ & CT & TT & T vs. C & TT + CT vs. CC & TT vs. $\mathrm{CC}+\mathrm{CT}$ & CT vs. CC & TT vs. CC \\
\hline \multicolumn{9}{|l|}{ Ethnicity } \\
\hline \multirow{2}{*}{$\begin{array}{l}\text { Caucasian } \\
\text { p value }\end{array}$} & $19 / 27$ & $49 / 109$ & $38 / 131$ & $0.63(0.45,0.88)$ & $0.52(0.27,0.97)$ & $0.58(0.36,0.92)$ & $0.64(0.32,1.26)$ & $0.41(0.21,0.82)$ \\
\hline & & & & 0.006 & 0.041 & 0.021 & 0.194 & 0.012 \\
\hline \multirow{2}{*}{$\begin{array}{l}\text { Asian } \\
\quad \text { p value }\end{array}$} & $224 / 264$ & $188 / 219$ & $32 / 44$ & $0.96(0.73,1.25)$ & $0.52(0.66,1.42)$ & $0.87(0.54,1.40)$ & $0.98(0.75,1.28)$ & $0.86(0.53,1.40)$ \\
\hline & & & & 0.750 & 0.887 & 0.575 & 0.904 & 0.548 \\
\hline
\end{tabular}

related with specific degradation of laminin and an increase in MMP-9 activity in experimental rat models. Similar results were reported by Guo et al. [35]. Both studies implied that MMP-9 activity was involved in the development of RGC apoptosis in glaucomatous rat eyes [35]. They also suggested that RGC apoptosis in glaucoma correlated strongly with elevated IOP and was significantly associated with IOP-induced changes in specific ECM [35]. These results suggested MMP-9 was strongly associated with glaucoma. SNPs rs17576 and rs3918249 are nonsynonymous single-base changes in corresponding coding regions; these changes increase the likelihood of their involvement in MMP-9 protein function. Taken together, the results from these studies suggest that $M M P$ 9 promoter polymorphisms (rs17576 and rs3918249) may be associated with glaucoma.

Two of the earliest studies on this subject investigated the relationship between the rs 17576 polymorphism and 


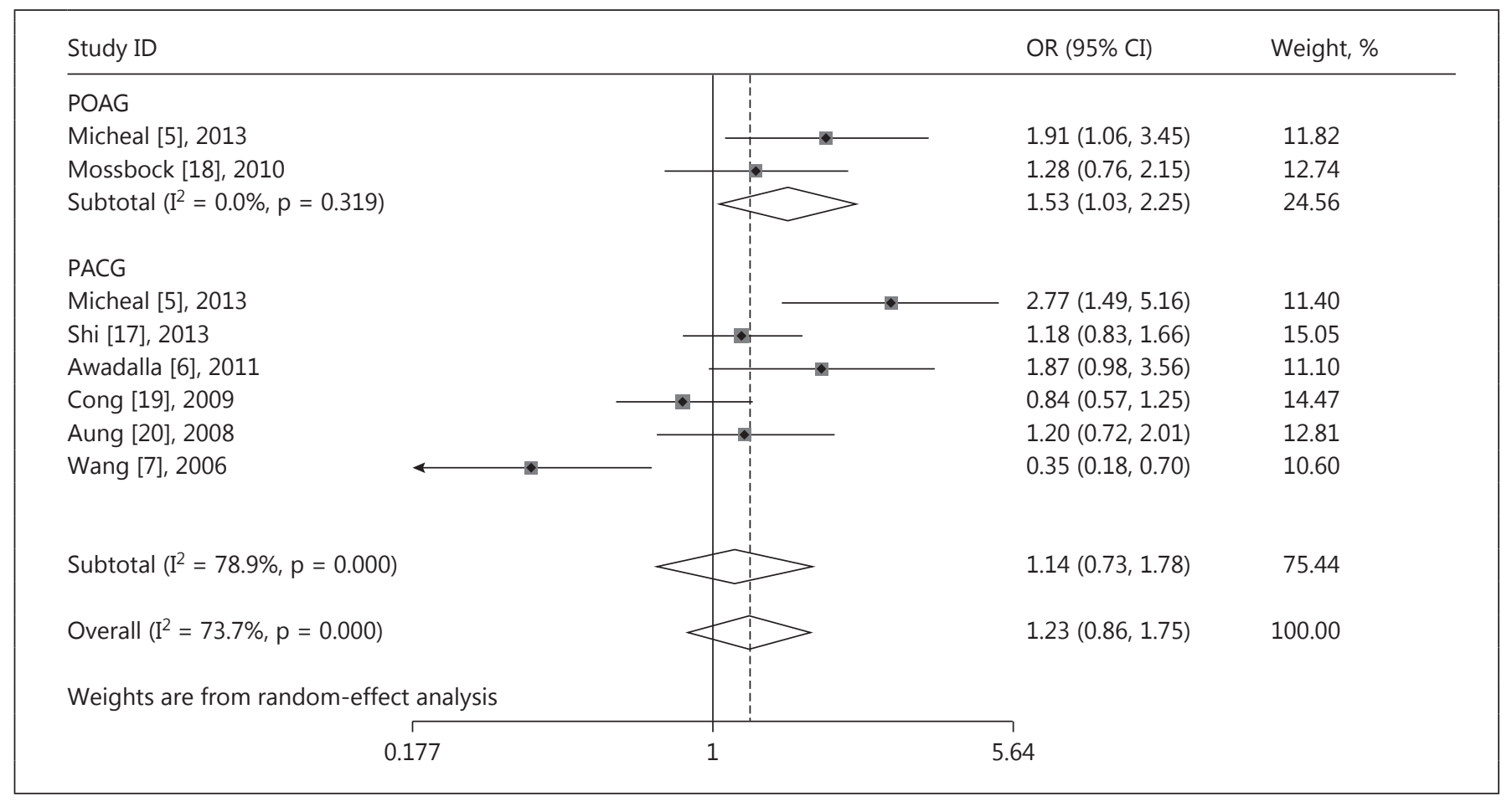

Fig. 3. Stratification analysis showing ORs for the association between the $M M P-9$ gene rs 17576 polymorphism and the risk of glaucoma (recessive model: GG vs. AA + AG).

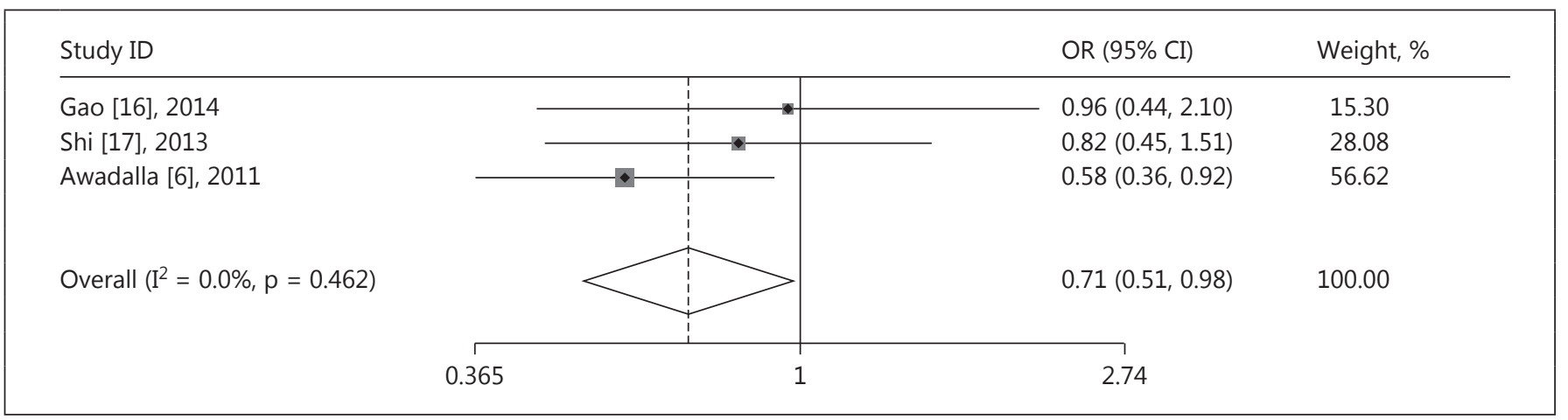

Fig. 4. Forest plot showing ORs for the association between the MMP-9 gene rs3918249 polymorphism and the risk of glaucoma (recessive model: TT vs. CC + CT).

PACG, and conflicting results were obtained with regard to Chinese ethnicity [7, 20]: Wang et al. [7] reported that rs 17576 was strongly associated with acute PACG in Taiwanese patients, but Aung et al. [20] failed to replicate this association in Singaporean Chinese subjects. They also evaluated chronic PACG but could not identify an association. It is noteworthy that the genotype frequencies of
PACG and the control subjects in the Singaporean study did not differ from those of patients with acute PACG in the Taiwanese study. However, a significant difference was observed with respect to the genotype frequencies of their control subjects [7,20]; this may explain the discrepancy in the results of these two studies. Two subsequent studies also failed to demonstrate an association between 
rs17576 and PACG in Chinese subjects [17, 19]. To investigate the association between rs17576 and glaucoma in Chinese ethnicity thoroughly, we conducted a meta-analysis on the 4 studies described above $[7,17,19,20]$. Our data suggested that rs17576 was not related to glaucoma in Chinese subjects, contradictory to the results of the Taiwanese study. The association between SNPs in genes with disorders is greatly affected by the number of subjects in the study. The Taiwanese study comprised 78 patients and 86 controls [7]; given the small number of participants, some caution is recommended with regard to the interpretation of results. Australian $[6,18]$ and Pakistani [5] studies showed that rs17576 was associated with glaucoma susceptibility. The gene functional polymorphism distribution among races may explain the conflicting findings between results based on Chinese ethnicity and those from other ethnicities. Regarding rs3918249, 3 studies investigated the relationship between this SNP and glaucoma, and results were not consistent $[6,16,17]$. The Australian study showed that rs3918249 was associated with glaucoma [6]; 2 Chinese studies failed to replicate this finding $[16,17]$. Our data suggested that rs3918249 might be a protective factor with regard to the onset of glaucoma, especially for Caucasian patients.

Several potential limitations are present in this metaanalysis. First, the sample size was not large, especially in stratification analyses. Second, some unpublished trials may have been missed, although our data indicated no evident publication bias. Third, our results were based on unadjusted estimates, without considering other confounders (such as age, gender or environmental factors); thus, a more precise analysis should be conducted, assuming the availability of individual data. Fourth, only Caucasian and Asian populations were included in this meta-analysis. Additional ethnic groups should be added to the study population due to ethnic differences in gene polymorphisms.

In conclusion, this meta-analysis did not demonstrate an association between rs17576 and the risk of glaucoma. Nevertheless, stratification analyses found that rs 17576 increased the risk of POAG. Our data also showed that rs3918249 was associated with a decreased risk of glaucoma, especially for Caucasian patients. However, largerscale studies are urgently needed to clarify the association between MMP-9 gene polymorphisms (rs17576 and rs3918249) and glaucoma to verify the present findings.

\section{Disclosure Statement}

The authors declare no conflict of interest.

\section{References}

1 Weinreb RN, Khaw PT: Primary open-angle glaucoma. Lancet 2004;363:1711-1720.

2 Quigley HA, Broman AT: The number of people with glaucoma worldwide in 2010 and 2020. Br J Ophthalmol 2006;90:262-267.

3 Worley A, Grimmer-Somers K: Risk factors for glaucoma: what do they really mean? Aust J Prim Health 2011;17:233-239.

4 Le A, Mukesh BN, McCarty CA, Taylor HR: Risk factors associated with the incidence of open-angle glaucoma: the visual impairment project. Invest Ophthalmol Vis Sci 2003;44: 3783-3789.

5 Micheal S, Yousaf S, Khan MI, Akhtar F, Islam F, Khan WA, den Hollander AI, Qamar $\mathrm{R}$, Ahmed A: Polymorphisms in matrix metalloproteinases MMP1 and MMP9 are associated with primary open-angle and angle closure glaucoma in a Pakistani population. Mol Vis 2013;19:441-447.

6 Awadalla MS, Burdon KP, Kuot A, Hewitt AW, Craig JE: Matrix metalloproteinase-9 genetic variation and primary angle closure glaucoma in a Caucasian population. Mol Vis 2011;17:1420-1424.
7 Wang IJ, Chiang TH, Shih YF, Lu SC, Lin LL, Shieh JW, Wang TH, Samples JR, Hung PT: The association of single nucleotide polymorphisms in the MMP-9 genes with susceptibility to acute primary angle closure glaucoma in Taiwanese patients. Mol Vis 2006;12:12231232.

8 St Jean PL, Zhang XC, Hart BK, Lamlum H, Webster MW, Steed DL, Henney AM, Ferrell RE: Characterization of a dinucleotide repeat in the $92 \mathrm{kDa}$ type IV collagenase gene (CLG4B), localization of CLG4B to chromosome 20 and the role of CLG4B in aortic aneurysmal disease. Ann Hum Genet 1995;59: $17-24$.

9 Wong TT, Sethi C, Daniels JT, Limb GA, Murphy G, Khaw PT: Matrix metalloproteinases in disease and repair processes in the anterior segment. Surv Ophthalmol 2002;47: 239-256.

10 Wiberg C, Heinegard D, Wenglen C, Timpl R, Morgelin M: Biglycan organizes collagen VI into hexagonal-like networks resembling tissue structures. J Biol Chem 2002;277:4912049126.
11 Lan J, Kumar RK, Di Girolamo N, McCluskey P, Wakefield D: Expression and distribution of matrix metalloproteinases and their inhibitors in the human iris and ciliary body. $\mathrm{Br} \mathrm{J}$ Ophthalmol 2003;87:208-211.

12 Hosseini M, Rose AY, Song K, Bohan C, Alexander JP, Kelley MJ, Acott TS: IL-1 and TNF induction of matrix metalloproteinase-3 by c-Jun $\mathrm{N}$-terminal kinase in trabecular meshwork. Invest Ophthalmol Vis Sci 2006; 47:1469-1476.

13 Babizhayev MA, Brodskaya MW: Fibronectin detection in drainage outflow system of human eyes in ageing and progression of openangle glaucoma. Mech Ageing Dev 1989;47: 145-157.

14 De Groef L, Van Hove I, Dekeyster E, Stalmans I, Moons L: MMPs in the trabecular meshwork: promising targets for future glaucoma therapies? Invest Ophthalmol Vis Sci 2013;54:7756-7763.

15 Acott TS, Kelley MJ: Extracellular matrix in the trabecular meshwork. Exp Eye Res 2008; 86:543-561. 
16 Gao XJ, Hou SP, Li PH: The association between matrix metalloprotease-9 gene polymorphisms and primary angle-closure glaucoma in a Chinese Han population. Int J Ophthalmol 2014;7:397-402.

17 Shi H, Zhu R, Hu N, Shi J, Zhang J, Jiang L, Jiang H, Guan H: Association of frizzled-related protein (MFRP) and heat shock protein 70 (HSP70) single nucleotide polymorphisms with primary angle closure in a Han Chinese population: Jiangsu Eye Study. Mol Vis 2013; 19:128-134.

18 Mossbock G, Weger M, Faschinger C, Zimmermann C, Schmut O, Renner W, El-Shabrawi $\mathrm{Y}$ : Role of functional single nucleotide polymorphisms of MMP1, MMP2, and MMP9 in open angle glaucomas. Mol Vis 2010;16:1764-1770.

19 Cong Y, Guo X, Liu X, Cao D, Jia X, Xiao X, Li S, Fang S, Zhang Q: Association of the single nucleotide polymorphisms in the extracellular matrix metalloprotease- 9 gene with PACG in southern China. Mol Vis 2009;15: 1412-1417.

20 Aung T, Yong VH, Lim MC, Venkataraman D, Toh JY, Chew PT, Vithana EN: Lack of association between the rs2664538 polymorphism in the MMP-9 gene and primary angle closure glaucoma in Singaporean subjects. J Glaucoma 2008;17:257-258.

21 Stang A: Critical evaluation of the NewcastleOttawa scale for the assessment of the quality of nonrandomized studies in meta-analyses. Eur J Epidemiol 2010;25:603-605.
22 Ownby RL, Crocco E, Acevedo A, John V, Loewenstein D: Depression and risk for Alzheimer disease: systematic review, meta-analysis, and metaregression analysis. Arch Gen Psychiatry 2006;63:530-538.

23 Higgins JP, Thompson SG: Quantifying heterogeneity in a meta-analysis. Stat Med 2002; 21:1539-1558.

24 Peters JL, Sutton AJ, Jones DR, Abrams KR, Rushton L: Comparison of two methods to detect publication bias in meta-analysis. JAMA 2006;295:676-680.

25 Lee NY, Park HY, Park CK, Ahn MD: Analysis of systemic endothelin-1, matrix metalloproteinase- 9 , macrophage chemoattractant protein-1, and high-sensitivity C-reactive protein in normal-tension glaucoma. Curr Eye Res 2012;37:1121-1126.

26 Markiewicz L, Majsterek I, Przybylowska K, Dziki L, Waszczyk M, Gacek M, Kaminska A, Szaflik J, Szaflik JP: Gene polymorphisms of the MMP1, MMP9, MMP12, IL-1 $\beta$ and TIMP1 and the risk of primary open-angle glaucoma. Acta Ophthalmol 2013;91:e516e523.

27 Sternlicht MD, Werb Z: How matrix metalloproteinases regulate cell behavior. Annu Rev Cell Dev Biol 2001;17:463-516.

28 Foster PJ, Baasanhu J, Alsbirk PH, Munkhbayar D, Uranchimeg D, Johnson GJ: Glaucoma in Mongolia. A population-based survey in Hövsgöl province, northern Mongolia. Arch Ophthalmol 1996;114:1235-1241.

29 Zhuang X, Zhu RR, Guan HJ, Huang CH, Shi WP, Jiang SY: A case-control study of factors associated with primary angle-closure glaucoma (in Chinese). Zhonghua Yan Ke Za Zhi 2008;44:503-506.
30 Abu-Amero KK, Morales J, Osman MN, Bosley TM: Nuclear and mitochondrial analysis of patients with primary angle-closure glaucoma. Invest Ophthalmol Vis Sci 2007;48: 5591-5596

31 Bradley JM, Vranka J, Colvis CM, Conger DM, Alexander JP, Fisk AS, Samples JR, Acott TS: Effect of matrix metalloproteinases activity on outflow in perfused human organ culture. Invest Ophthalmol Vis Sci 1998;39: 2649-2658.

32 Maatta M, Tervahartiala T, Harju M, Airaksinen J, Autio-Harmainen H, Sorsa T: Matrix metalloproteinases and their tissue inhibitors in aqueous humor of patients with primary open-angle glaucoma, exfoliation syndrome, and exfoliation glaucoma. J Glaucoma 2005; 14:64-69.

33 De Groef L, Van Hove I, Dekeyster E, Stalmans I, Moons L: MMPs in the neuroretina and optic nerve: modulators of glaucoma pathogenesis and repair? Invest Ophthalmol Vis Sci 2014;55:1953-1964.

34 Chintala SK, Zhang X, Austin JS, Fini ME: Deficiency in matrix metalloproteinase gelatinase B (MMP-9) protects against retinal ganglion cell death after optic nerve ligation. J Biol Chem 2002;277:47461-47468.

35 Guo L, Moss SE, Alexander RA, Ali RR, Fitzke FW, Cordeiro MF: Retinal ganglion cell apoptosis in glaucoma is related to intraocular pressure and IOP-induced effects on extracellular matrix. Invest Ophthalmol Vis Sci 2005; 46:175-182. 